\title{
FIXAÇÃO DE NITROGÊNIO: ESTRUTURA, FUNÇÃO E MODELAGEM BIOINORGÂNICA DAS NITROGENASES
}

\author{
Fábio Souza Nunes*, Angela Cristina Raimondi e Antonio Carlos Niedwieski \\ Departamento de Química, Universidade Federal do Paraná, CP 19081, 81531-990 Curitiba - PR
}

Recebido em 15/10/02; aceito em 10/3/03

\begin{abstract}
NITROGEN FIXATION: STRUCTURE, FUNCTION AND BIOINORGANIC MODELING OF THE NITROGENASES. Biological nitrogen fixation, catalyzed by nitrogenases, contributes about half of the nitrogen needed to global agriculture. For forty years synthetic chemists and theoreticians have tried to understand and model the structure and function of this important metalloenzyme. Ten years after the first report on the crystal structure of the MoFe protein, scientists still have not been able to synthesize a chemical equivalent of the FeMo cofactor nor the structure knowledge revealed the key to its catalytic activity. This paper with 104 references presents a review of the most relevant advances in chemical nitrogen fixation and their relation with the nitrogenases.
\end{abstract}

Keywords: nitrogenase; dinitrogen complexes; iron-molybdenum cofactor.

\section{INTRODUÇÃO}

Dinitrogênio $\left(\mathrm{N}_{2}\right)$ é o composto mais abundante que contém nitrogênio e, ao mesmo tempo, é o menos reativo deles. É uma fonte atrativa para a obtenção de compostos nitrogenados necessários na indústria, como a amônia, empregada na produção industrial de fertilizantes, explosivos, plásticos e vernizes.

Em $1886^{1}$ provou-se empiricamente a fixação biológica de nitrogênio, ou seja, a conversão de nitrogênio gasoso em outras espécies químicas nitrogenadas promovida por alguns organismos denominados diazótrofos, que empregam o nitrogênio fixado na biossíntese de proteínas e ácidos nucléicos². Em 1913, Haber e Bosch implantaram um processo industrial capaz de converter $\mathrm{o}_{2}$ gasoso em amônia. Este processo de fixação química de nitrogênio emprega ferro metálico como catalisador e depende de altas temperaturas $\left(\approx 500^{\circ} \mathrm{C}\right)$ e pressões (200-600 atm) para combinar $\mathrm{N}_{2}$ e $\mathrm{H}_{2}$ produzindo amônia em grande escala ${ }^{3}$. As condições drásticas e a necessidade de um catalisador no processo Haber-Bosch são exigidas devido à alta estabilidade da molécula de $\mathrm{N}_{2}$ em condições ambientes. Apesar da reação envolvida ser exotérmica e termodinamicamente favorecida, a quebra da ligação $\mathrm{N} \equiv \mathrm{N}$ requer uma energia de ativação bastante alta $(1035 \mathrm{~kJ} / \mathrm{mol})^{4}$. $\mathrm{O}$ mecanismo de formação da amônia no processo de Haber-Bosch ainda é desconhecido, embora seja evidente que a etapa limitante do processo seja a quebra da tripla ligação, $\mathrm{N} \equiv \mathrm{N}$, formando íons nitretos, $\mathrm{N}^{3-}$.

As condições amenas presentes na fixação biológica $\left(20{ }^{\circ} \mathrm{C}\right.$, $0,8 \mathrm{~atm}$, potencial redox de $\sim 500 \mathrm{mV})^{4} \mathrm{em}$ contraste àquelas empregadas no processo Haber-Bosch estimularam a busca do agente biológico responsável pela catálise da ativação e fixação do nitrogênio ${ }^{5}$. No período de 1930-1936, Bortels ${ }^{6}$ descreveu que o molibdênio e, numa menor extensão, o vanádio eram necessários para a fixação biológica de dinitrogênio atmosférico por bactérias do solo. $\mathrm{O}$ conjunto de enzimas responsável pela fixação de $\mathrm{N}_{2}$ em meio biológico foi denominado nitrogenase.

As descobertas que se seguiram revelaram que, independente do tipo de organismo fixador de nitrogênio do qual são isoladas, as nitrogenases são enzimas compostas por duas metaloproteínas, as quais

*e-mail: fsnunes@quimica.ufpr.br possuem propriedades e requerimentos similares para atividade, como a ausência de dioxigênio, uma fonte de redutores de baixo potencial redox e a hidrólise de MgATP (ATP = adenosina 5'-trifosfato).

Uma pesquisa detalhada sobre os mecanismos da fixação de $\mathrm{N}_{2}$ começou no início da década de $60 \mathrm{com}$ a primeira preparação reprodutível de extratos ativos de células na catálise da redução de $\mathrm{N}_{2}^{1,7}$. Já em 1964, os químicos interessaram-se pelo problema, impulsionados pela descoberta de Vol'pin de que a hidrólise de alguns complexos organometálicos em baixo estado de oxidação produziam quantidades significativas de amônia ${ }^{8}$. Em 1965, Allen e Senoff ${ }^{9}$ publicaram a descoberta do primeiro complexo sintético de dinitrogênio, $\left[\mathrm{Ru}\left(\mathrm{NH}_{3}\right)_{5} \mathrm{~N}_{2}\right]^{2+}$.

Hoje, a pesquisa sobre a fixação de nitrogênio acumula 40 anos e a busca pela fonte de fertilizantes de baixo custo continua. Conhecemos a estrutura da nitrogenase de molibdênio com grande detalhe ${ }^{4}$, mas o mecanismo a nível molecular da fixação de $\mathrm{N}_{2}$ está longe de ser completamente compreendido.

\section{A ESTRUTURA E O MODO DE AÇÃO DA NITROGENASE DE MOLIBDÊNIO}

A nitrogenase de molibdênio consiste de uma ferro-proteína (Feproteína) e de uma molibdênio-ferro-proteína (MoFe-proteína). A Fe-proteína funciona como doadora de elétrons para a MoFe-proteína (que contém o cofator da enzima, FeMoco), num processo dependente de hidrólise de MgATP.

Em 1992, a primeira estrutura de raios-X (com resolução de 2,8 $\AA$ ) foi descrita por Kim e Rees ${ }^{2}$, com sucessivos melhoramentos na resolução em $1997(2,0 \AA)^{2}$ e em $1999(1,6 \AA)^{2}$. A determinação da estrutura cristalográfica da MoFe-proteína permitiu uma visão mais detalhada da constituição da enzima e de seu provável sítio ativo $^{4,10}$. A Fe-proteína é um dímero de subunidades idênticas, com uma massa molar total de $\sim 60 \mathrm{kDa}$. As duas subunidades estão conectadas covalentemente por um agregado [4Fe-4S], e contêm, cada uma, um sítio de ligação de nucleotídeos (MgATP e MgADP). Aparentemente, existe uma modulação alostérica no acoplamento do nucleotídeo e o comportamento redox do agregado metálico, envolvendo dois (ou mais) estados conformacionais da Fe-proteína.

A MoFe-proteína existe como um tetrâmero $\alpha_{2} \beta_{2}$ com massa molar total de $\sim 240 \mathrm{kDa}$ e possui quatro centros redox: dois cofatores 
FeMoco (Figura 1a) localizados nas subunidades $\alpha$ (alojados a $10 \AA$ sob a superfície da proteína) e dois clusters $\mathrm{P}$ de composição [8Fe:7S], cada um constituído por dois cubanos [4Fe-3S] ligados em ponte por um átomo de enxofre hexacoordenado. Os clusters P estão situados na interface das subunidades $\alpha$ e $\beta$, também a cerca de $10 \AA$ abaixo da superfície da proteína ${ }^{4}$ e, ligados à cadeia protéica através de resíduos de cisteína.

Duas possíveis valências foram propostas para os íons metálicos no cofator FeMoco para a enzima no seu "resting state": $\mathrm{Mo}^{\mathrm{IV}} 6 \mathrm{Fe}^{\mathrm{II}}$ $\mathrm{Fe}^{\mathrm{III}}$ e $\mathrm{Mo}^{\mathrm{IV}} 4 \mathrm{Fe}^{\mathrm{II}} 3 \mathrm{Fe}^{\mathrm{III}}{ }^{11}$.

A geometria não usual (trigonal prismática) dos seis átomos centrais de ferro é, talvez, a característica mais marcante do cofator. Estes átomos estão na superfície de uma esfera de raio de $2,0 \AA$ do centro do cofator e estão ligados individualmente a três átomos de enxofre. Por sua vez, os nove átomos de enxofre estão equidistantes do centro de uma segunda esfera, de raio igual a 3,3 $\AA^{12}$.

Dez anos após a primeira determinação estrutural da nitrogenase uma análise cristalográfica de alta resolução $(1,16 \AA$ ) revelou segredos ainda desconhecidos sobre o cofator $\mathrm{FeMoco}^{12}$. Os resultados mostraram a existência de um ligante coordenado a seis átomos de ferro no centro do cofator (Figura 1b). A densidade eletrônica deste ligante é mascarada em estruturas com resolução maior que $1,55 \AA$. O refinamento cristalográfico a $1,16 \AA$ é consistente com um ligante contendo um átomo doador leve, possivelmente nitrogênio. A biossíntese do cofator é complexa e não completamente conhecida, mas acredita-se que o papel deste nitrogênio intersticial possa estar relacionado com sua estabilização ${ }^{13}$. Com esta recente descoberta, pode-se afirmar que a geometria em torno dos átomos de ferro é, portanto, tetraédrica e não trigonal prismática como indicado pelas estruturas anteriores.

A formação do complexo entre a Fe-proteína e a MoFe-proteína é uma etapa essencial à catálise de reações realizadas pela nitrogenase ${ }^{14}$. Acredita-se que a redução biológica de $\mathrm{N}_{2}$ processa-se em três etapas ${ }^{4}$ : 1) a redução da Fe-proteína por ferredoxinas ou flavodoxinas, 2) a transferência multieletrônica da Fe-proteína para a Mo-Fe proteína, (em que um elétron é transferido de cada vez) acoplada à hidrólise de até duas moléculas de MgATP por elétron transferido ${ }^{4,15,16}$ e 3) a redução propriamente dita do substrato no sítio ativo da MoFe-proteína.

Sob condições ótimas, a redução de $\mathrm{N}_{2}$ catalisada pela nitrogenase de molibdênio pode ser representada pela equação ${ }^{1}$ :

$\mathrm{N}_{2}+8 \mathrm{e}^{-}+8 \mathrm{H}^{+}+16 \mathrm{MgATP} \rightarrow 2 \mathrm{NH}_{3}+\mathrm{H}_{2}+16 \mathrm{MgADP}+16 \mathrm{P}_{\mathrm{i}}$

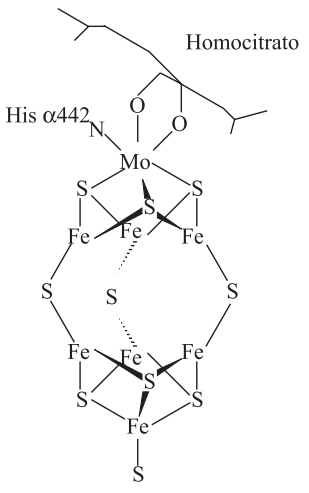

Cis $\alpha 275$

\section{a}

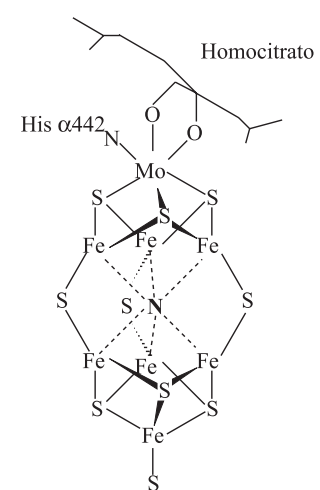

Cis $\alpha 275$
Figura 1. Representação esquemática do modelo do cofator FeMoco da nitrogenase: (a) com resolução maior que $1.55 \AA$ e (b) com resolução de $1.16 \AA$, revelando a presença de um átomo intersticial leve, possivelmente nitrogênio

\section{AS NITROGENASES ALTERNATIVAS}

Contrariando as considerações históricas sobre a importância essencial do molibdênio para o funcionamento das nitrogenases ${ }^{17}$, ainda na década de 80 descobriu-se que várias classes de bactérias são capazes de fixar nitrogênio em condições de deficiência de molibdênio, utilizando sistemas enzimáticos alternativos, como a nitrogenase de vanádio e ferro (nitrogenase $\mathrm{VFe})^{10,18}$, ou a que contém apenas ferro ${ }^{10}$ como metal de transição (nitrogenase $\mathrm{Fe}$ ). Um quarto tipo de nitrogenase foi recentemente caracterizado na bactéria termofílica Streptomyces thermoautotrophicus, cuja propriedade mais notável é a dependência de oxigênio e do radical superóxido ambos nocivos para a maioria das nitrogenases. O nitrogênio é fixado segundo a equação: $\mathrm{N}_{2}+8 \mathrm{e}^{-}+8 \mathrm{H}^{+}+4-12 \mathrm{MgATP} \rightarrow 2 \mathrm{NH}_{3}+\mathrm{H}_{2}$ + 4-12MgADP + 4-12 $\mathrm{PO}_{4}^{3-}$. A reação é acoplada à oxidação de monóxido de carbono realizada por uma enzima do tipo desidrogenase, que contém molibdênio. Esta enzima usa os elétrons da oxidação do $\mathrm{CO}$ para reduzir oxigênio até radicais superóxidos que, por sua vez, são reoxidados $\mathrm{a}_{2}$ por uma enzima superóxido óxidoredutase, que contém manganês. Os elétrons são finalmente transferidos para uma MoFe-nitrogenase, que reduz nitrogênio até amônia ${ }^{19}$.

Antes da determinação da estrutura cristalina da nitrogenase de molibdênio, estudos de EXAFS forneceram importantes detalhes sobre o ambiente químico do molibdênio na Mo-proteína ${ }^{4}$. As atribuições baseadas nesta técnica foram confirmadas pela estrutura de raios-X de cristal.

Os centros redox presentes na nitrogenase de vanádio foram investigados por EPR, MCD (Dicroísmo Circular Magnético), Mössbauer e espectroscopias de absorção de raios-X, como EXAFS, mas não se dispõe ainda de estruturas de raios- $X$ de cristal desta e da nitrogenase contendo apenas ferro ${ }^{10}$.

Na nitrogenase de vanádio observa-se um terceiro tipo de subunidade além da $\alpha$ e $\beta$; a subunidade $\delta$ é componente essencial para estabilizar a estrutura quaternária hexamérica $\alpha_{2} \beta_{2} \delta_{2}$. Dados espectroscópicos indicam $\mathrm{V}^{\mathrm{II}}$ ou $\mathrm{V}^{\mathrm{IV}}$ numa geometria octaédrica distorcida no cofator $\mathrm{FeVco}^{10,18,21}$. Na realidade, foi possível extrair o cofator ferro-vanádio das VFe-proteínas, na forma de um agregado de átomos contendo $\mathrm{V}, \mathrm{Fe}$ e $\mathrm{S}$ numa estequiometria aproximadamente igual a $\mathrm{VFe}_{5-7} \mathrm{~S}_{4-6}{ }^{10,17}$.

Sob condições ótimas a $30{ }^{\circ} \mathrm{C}$ e em presença de quantidade saturante de $\mathrm{N}_{2}$, a vanádio-nitrogenase catalisa a redução de $\mathrm{N}_{2}$ segundo a equação ${ }^{18}$ :

$\mathrm{N}_{2}+12 \mathrm{e}^{-}+12 \mathrm{H}^{+}+24 \mathrm{MgATP} \rightarrow 2 \mathrm{NH}_{3}+3 \mathrm{H}_{2}+24 \mathrm{MgADP}+24 \mathrm{P}_{\mathrm{i}}$

Os estudos espectroscópicos da proteína que contém apenas ferro estão limitados à espectroscopia de EPR, que tem sido usada para identificar os tipos de centro redox ${ }^{20}$.

Nas três classes de enzima (Mo, V ou Fe) observa-se a conservação dos resíduos de aminoácidos, aos quais se ligam os centros metálicos, sugerindo a presença de centros redox homólogos ${ }^{10}$.

\section{A FORMAÇÃO DE $\mathrm{H}_{2}$ E A EFICIÊNCIA DAS NITROGENASES}

Prótons competem com $\mathrm{N}_{2}$ como substrato passível de redução pelas nitrogenases. Esta competição diminui a eficiência das enzimas. No caso da Mo-nitrogenase, um máximo de $25 \%$ dos elétrons que fluem pelo sistema são utilizados para a produção de $\mathrm{H}_{2}$, contra até $50 \%$ no que diz respeito à vanádio-nitrogenase ${ }^{17,18,22}$. A evolução de hidrogênio é espontânea e não depende da ligação de qualquer outro substrato para acontecer.

A formação de HD pela nitrogenase de molibdênio quando da redução de $\mathrm{N}_{2}$, na presença de $\mathrm{D}_{2}$, é um dos fenômenos mais interes- 
santes e ainda não completamente compreendido, associado à fixação biológica de nitrogênio. $\mathrm{O}$ fenômeno está associado exclusivamente à redução de $\mathrm{N}_{2}$. A formação de $\mathrm{HD}$ requer que a molécula de $\mathrm{D}_{2}$ sofra redução no sítio ativo da enzima, de acordo com a equação: $\mathrm{D}_{2}+2 \mathrm{H}^{+}$ $+2 \mathrm{e}^{-} \rightarrow 2 \mathrm{HD}$. Não existem evidências da inserção de deutério nas moléculas de amônia, sugerindo que a formação de HD e a redução de $\mathrm{N}_{2}$ ocorrem em sítios diferentes no cofator $\mathrm{FeMoco}^{23}$. Uma racionalização possível é a ligação de $\mathrm{N}_{2}$ ao átomo de molibdênio, por exemplo, ativando o sítio de ferro para a coordenação de $\mathrm{D}_{2}$, que reagiria com um fluxo de elétrons dos clusters $\mathrm{P}$ e com prótons do solvente para formar HD. Em uma segunda explicação, acredita-se que o sítio de ligação de $\mathrm{N}_{2}$ no FeMoco seja formado por triidretos. Com a liberação de $\mathrm{H}_{2}$ acoplada à redução de $\mathrm{N}_{2}$ até $\mathrm{NH}_{3} \mathrm{o}$ sítio ativo teria $\mathrm{D}_{2}$ na presença de hidretos, favorecendo a formação de $\mathrm{HD}^{23}$. Reatividade semelhante foi observada em modelos bioinorgânicos como o $\left[\mathrm{CoH}_{3}\left(\mathrm{PPh}_{3}\right)_{3}\right]^{24}$. Neste contexto, é sabido que triidreto-complexos de ferro como $\left[\mathrm{FeH}_{3}(\mathrm{dmpe})_{2}\right]^{+}\left(\mathrm{dmpe}=\mathrm{Me}_{2} \mathrm{PCH}_{2} \mathrm{CH}_{2} \mathrm{PMe}_{2}\right)$ reagem com doadores, como MeCN, liberando $\mathrm{H}_{2}$ e formando $[\mathrm{FeH}(\mathrm{MeCN})(\mathrm{dmpe})]^{+}{ }^{23}$.

\section{O MODO DE LIGAÇÃO DO N 2 NO SÍTIO ATIVO DAS NITROGENASES}

Apesar do grande avanço após a caracterização estrutural da nitrogenase de molibdênio, ainda existem muitas dúvidas sobre o seu mecanismo de ação. O modo eficiente com que a enzima controla o fluxo de íons hidrogênio e de elétrons de forma catalítica ainda é alvo de várias especulações e hipóteses. Outra questão situa-se no modo como o $\mathrm{N}_{2}$ se liga ao cofator da nitrogenase. Não há resposta definitiva para esta questão, mas praticamente todos os modelos da nitrogenase assumem a manutenção da estrutura fechada do cofator FeMoco, determinada para o "resting state" da enzima. Acredita-se que a ativação da molécula de $\mathrm{N}_{2}$ ocorra, por exemplo, a) via ligação a seis átomos de ferro, dentro da gaiola do cofator; b) do lado de fora da gaiola, através da coordenação a quatro centros de ferro; ou c) através da ligação ao átomo de molibdênio, após a labilização do ligante homocitrato (Figura 2).
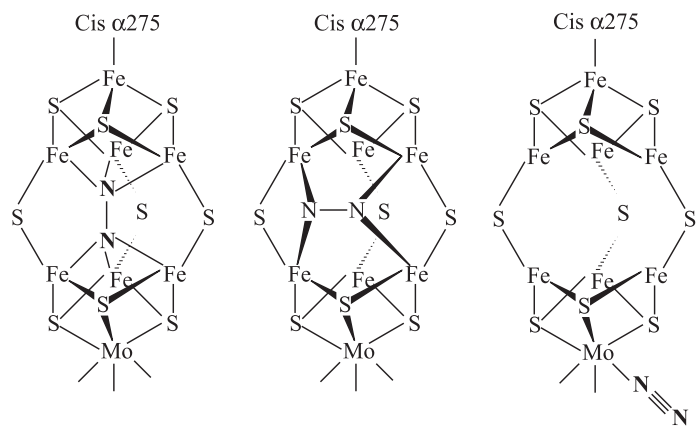

Figura 2. Representação esquemática dos modos de ligação do $\mathrm{N}_{2}$ no cofator FeMoco

A ligação aos seis átomos de ferro nos faz pensar se o nitrogênio intersticial, descoberto por Rees não estaria associado ao processo de fixação ou seria ele parte de um resíduo de aminoácido? A ligação via átomo de ferro é justificada pelo fato deste metal encontrarse presente em todos os tipos de nitrogenases. Além disso, a geometria trigonal não usual apresentada pelos seis sítios de ferro centrais no cofator FeMoco induz à idéia de que esta insaturação na esfera de coordenação poderia ser relevante para a ligação de substratos e outros ligantes ${ }^{3,21}$. Acredita-se ser pouco provável que diferentes substratos satisfaçam igualmente os requisitos estéreos do sítio ativo da nitrogenase. A ligação a múltiplos centros de ferro no cofator eliminaria este problema. As propriedades espectrais da nitrogenase da Klebsiella pneumoniae indicam que ligantes como fenilselenol, tiofenol e 2-bromotiofenol se ligam a átomos de ferro do cofator $\mathrm{FeMoco}^{21}$. Por outro lado, a rica química de compostos modelo da nitrogenase que utilizam molibdênio ou metais correlatos como vanádio, aponta fortemente para a participação destes metais na ligação e no mecanismo de redução de $\mathrm{N}_{2}^{21,22,26,27}$.

\section{A MODELAGEM DAS NITROGENASES}

A formação de amônia a partir de $\mathrm{H}_{2}$ e $\mathrm{N}_{2}$ é termodinamicamente favorável $\left(\Delta \mathrm{G}^{\mathrm{o}}=-31,8 \mathrm{~kJ} / \mathrm{mol} \text { a } 25^{\circ} \mathrm{C} \mathrm{e} 1 \mathrm{~atm}\right)^{4}$. O processo torna-se não espontâneo a aproximadamente $190{ }^{\circ} \mathrm{C}$, enquanto a quebra da ligação tripla $\mathrm{N}-\mathrm{N}$ ocorre a temperaturas muito mais elevadas $\left(\sim 3200^{\circ} \mathrm{C}\right)$. As altas temperaturas são minimizadas com o uso de altas pressões e catalisadores. $\mathrm{O}$ processo industrial está tão bem otimizado que não há interesse no desenvolvimento de condições alternativas mais brandas. Apesar do apelo econômico relacionado à grande disponibilidade de $\mathrm{N}_{2}$ atmosférico e à importância industrial da amônia, o interesse em compreender o funcionamento da nitrogenase tem sido exclusivamente acadêmico.

A fixação biológica de nitrogênio corresponde, hoje, a cerca da metade de toda a fonte de nitrogênio para a agricultura mundial, o restante vem de fertilizantes produzidos quimicamente através da amônia do processo Haber-Bosch ${ }^{13}$. A produção de amônia é a segunda no mundo, perdendo apenas para o $\mathrm{H}_{2} \mathrm{SO}_{4}$. A redução da molécula de $\mathrm{N}_{2}$ até amônia requer seis elétrons. Embora este processo seja termodinamicamente favorável $\left(\Delta \mathrm{G}^{\circ}=-62,7 \mathrm{~kJ} / \mathrm{mol}, 25^{\circ} \mathrm{C}, \mathrm{pH}=7\right)$, ele é complicado devido à estabilidade cinética da molécula de $\mathrm{N}_{2}$. Existe uma grande barreira energética associada à quebra da ligação tripla. Comparando-se as energias das ligações tripla, dupla e simples na molécula de $\mathrm{N}_{2}, 929,413$ e $161 \mathrm{~kJ} / \mathrm{mol}$, respectivamente, observase que a energia necessária para a quebra da ligação tripla corresponde a mais de $50 \%$ da energia total de ligação da molécula ${ }^{26}$. Outra dificuldade está na quebra da ligação M-N de um nitreto-, hidrazido- ou amidetocomplexo, justificando o uso comum de agentes redutores muito fortes (como sais do grupo principal ${ }^{22}$ ou amálgama de sódio $^{28}$ ) na modelagem da função da enzima. O lítio é o único elemento do grupo principal capaz de reduzir $\mathrm{N}_{2}$ em condições ambientes.

Metais de transição em baixo estado de oxidação com menor poder redutor, freqüentemente, demonstram capacidade para interagir com dinitrogênio $5,26,27,29$. Embora o potencial de redução não seja comparável ao do metal alcalino, a retrodoação torna a molécula de $\mathrm{N}_{2}$ mais susceptível à dissociação, mostrando que a coordenação de $\mathrm{N}_{2}$ é pré-requisito essencial para as transformações subseqüentes.

A eficiência do sistema biológico tem inspirado químicos sintéticos a preparar e caracterizar complexos metálicos com estruturas moleculares muito próximas àquela do cofator da nitrogenase ${ }^{30}$. Entretanto, apesar da grande semelhança estrutural, até hoje, nenhum dos modelos estruturais mostrou atividade para a redução de $\mathrm{N}_{2}(\mathrm{~g})$ ou para estabilizar um complexo de dinitrogênio.

Passados dez anos desde a resolução da estrutura de raios-X da nitrogenase de molibdênio, ficou evidente que o domínio estrutural não garantiu o conhecimento da função da enzima, nem tão pouco revelou o procedimento para a síntese de catalisadores competitivos. A complexidade estrutural da nitrogenase ${ }^{4}$, associada ao fato de que a atividade catalítica da enzima é suportada pela parte protéica (cofatores isolados não catalisam a redução de $\mathrm{N}_{2}$ ), faz com que a grande maioria dos estudos concentrem-se em modelos funcionais (e não estruturais) da metaloproteína, basicamente complexos de metais de transição de baixa massa molar.

A necessidade de íons de metais de transição na fixação de nitrogênio sugeriu que complexos metálicos contendo dinitrogênio, análo- 
gos aos complexos carbonilo já conhecidos $\left(\mathrm{CO}\right.$ e $\mathrm{N}_{2}$ são isoeletrônicos), fossem bons modelos. Grande parte dos compostosmodelo descritos ao longo dos anos compõe basicamente três classes: ${ }^{7}$ a maior delas é constituída por complexos que contém $\mathrm{N}_{2}$ ligado ao metal na forma terminal ( $\left.\mathrm{M}-\mathrm{N}_{2}\right)$, em que o comprimento da ligação $\mathrm{N}-\mathrm{N}$ não é muito maior que o da molécula livre e há pequena evidência de ativação ${ }^{31,32}$. A segunda classe contém $\mathrm{N}_{2}$ terminal, mas ligando dois átomos metálicos em ponte, com distâncias $\mathrm{N}-\mathrm{N}$ muito próximas às do $\mathrm{N}_{2}$ livre $(\mathrm{M}-\mathrm{N} \equiv \mathrm{N}-\mathrm{M})^{58}$. A terceira classe também contém $\mathrm{N}_{2}$ ligado em ponte mas, neste caso, a distância N-N é consideravelmente maior que a da forma livre $(\mathrm{M}=\mathrm{N}-\mathrm{N}=\mathrm{M})^{54}$. O objetivo final é ativar a molécula de nitrogênio. $\mathrm{O}$ trabalho pioneiro de Chat $\mathrm{t}^{25}$ sobre a preparação de complexos de molibdênio e tungstênio contendo $\mathrm{N}_{2}$ representa bem os esforços nesta área. Chatt descreveu, pela primeira vez, a redução de $\mathrm{N}_{2}$ em meio prótico por complexos do tipo $\left[\mathrm{M}\left(\mathrm{N}_{2}\right)\left(\mathrm{PR}_{3}\right)_{4}\right]$ $\left(\mathrm{M}=\mathrm{Mo}\right.$ ou $\mathrm{W}$ ) em uma relação $\mathrm{N}_{2} / \mathrm{M}$ de 1:125 (Figura 3). Apesar da possibilidade de síntese de compostos contendo $\mathrm{N}_{2}$ coordenado, há uma enorme dificuldade em garantir uma natureza catalítica ao processo de redução. Numa seqüência de protonações do tipo $\mathrm{M}-\mathrm{N}_{2}$, M$\mathrm{N}=\mathrm{N}-\mathrm{H}, \mathrm{M}=\mathrm{N}-\mathrm{NH}_{2}, \mathrm{M} \equiv \mathrm{N}-\mathrm{NH}_{3}{ }^{7}$, o principal obstáculo é manter um fluxo contínuo de seis elétrons originados do metal, provocando a sua oxidação, acoplado ao fornecimento de íons $\mathrm{H}^{+}$e, ao mesmo tempo, assegurar a integridade da ligação $\mathrm{M}-\mathrm{N}_{2}$, a estabilidade das espécies intermediárias e a regeneração do metal à sua forma reduzida no final do processo ${ }^{11}$. Apesar de se poder manter um suprimento de íons hidrogênio através de um ácido mineral, são raros os sistemas-modelo descritos até hoje que apresentam este tipo de propriedade de forma catalítica. Pelo contrário, a grande maioria dos sistemas-modelo age de forma estequiométrica e não cíclica.

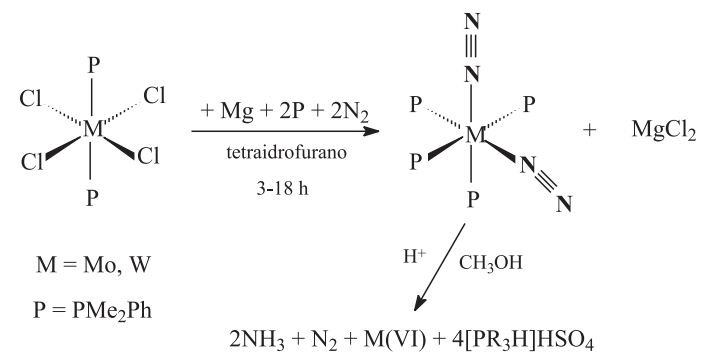

Figura 3. Redução de dinitrogênio coordenado a complexos-modelo de Chatt em meio prótico

\section{OS MODELOS QUÍMICOS PARA A FUNÇÃO DAS NITROGENASES}

A polinuclearidade e o baixo estado de oxidação dos metais no sítio ativo da nitrogenase parecem ser fatores importantes no processo de ativação e redução do dinitrogênio ${ }^{26}$. O cofator FeMoco funciona, aparentemente, como uma fonte de elétrons onde os metais interagem através de ligações em ponte.

Vários complexos-modelo de molibdênio ${ }^{22,26,33-36}$, vanádio ${ }^{26,27,37-45}$, ferro $^{23,33,40,45-47}$, titânio ${ }^{48}$, nióbio ${ }^{49,50}$, zircônio ${ }^{5,51}$ e outros metais, como tungstênio ${ }^{35}$, rutênio ${ }^{23,52,53}$, ósmio ${ }^{23}$, cobalto ${ }^{45}$, ouro ${ }^{29,54}$ e samário ${ }^{55}$ foram sintetizados e caracterizados. Em alguns destes estudos, a atividade é catalítica ${ }^{26,37,41,48}$ para produção de amônia. Um dos modelos mais eficientes é aquele de molibdênio(III) ${ }^{22}$. Os complexos de $\mathrm{Mo}^{\mathrm{III}}$ catalisam a redução de $\mathrm{N}_{2}$ apenas na presença de redutores fortes, como uma mistura de hidróxidos de $\mathrm{Ti}(\mathrm{OH})_{3}$ e $\mathrm{Mg}(\mathrm{OH})_{2}$ ou de amálgama de sódio. Para entender melhor o papel único do molibdênio(III) nestes sistemas, recorremos ao recente trabalho de Cummins $^{34}$ que descreveu a síntese do complexo $\left\{\mathrm{Mo}\left[\mathrm{NBu}^{\mathrm{t}}\right.\right.$ $\left.\left.\left(\mathrm{C}_{6} \mathrm{H}_{3} \mathrm{Me}_{2}\right)\right]_{3}\right\}$ e a quebra, passo a passo, da tripla ligação do $\mathrm{N}_{2}$, formando o nitreto complexo, $\left\{\mathrm{N} \equiv \mathrm{Mo}\left[\mathrm{NBu}^{\mathrm{t}}\left(\mathrm{C}_{6} \mathrm{H}_{3} \mathrm{Me}_{2}\right)\right]_{3}\right\}$. Para este sistema, supõe-se que a quebra da ligação tripla se dê pela formação de uma ligação em ponte ("end on") do $\mathrm{N}_{2}$ a dois centros de molibdênio (Figura 4). A reação é movida termodinamicamente pela estabilidade elevada da ligação $\mathrm{Mo} \equiv \mathrm{N}$, enquanto que a configuração eletrônica $\mathrm{d}^{3}$ permite a transferência de seis elétrons para o $\mathrm{N}_{2}$ coordenado. Resultados semelhantes também foram descritos por Gambarotta para vanádio ${ }^{42}$ e nióbio ${ }^{49}$.

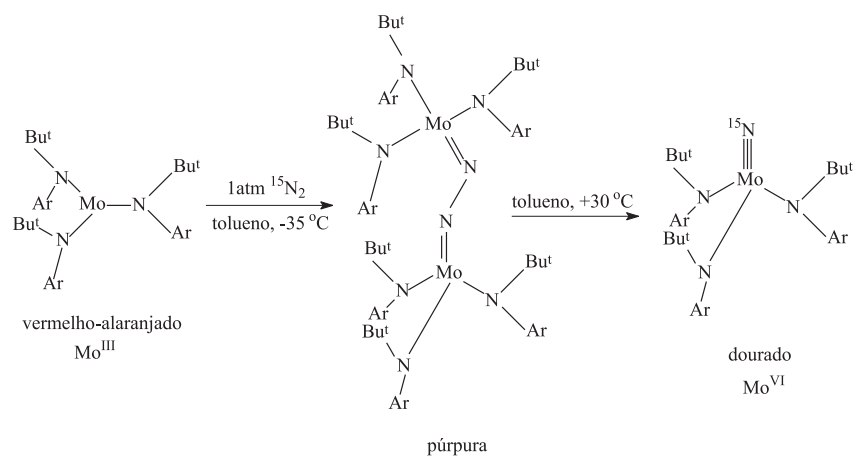

Figura 4. Seqüência de reações proposta por Cummins para a quebra da tripla ligação do $\mathrm{N}_{2}$ no amideto-complexo [ $\left.\mathrm{Mo}\left(\mathrm{NBu}^{t} \mathrm{Ar}\right)_{3}\right]\left(\mathrm{Bu}^{t}=-\mathrm{C}\left(\mathrm{CD}_{3}\right)_{2} \mathrm{CH}_{3}\right.$ e $\mathrm{Ar}=3,5-\mathrm{C}_{6} \mathrm{H}_{3} \mathrm{Me}_{2}$ ) formando o nitreto-complexo $\mathrm{N} \equiv \mathrm{Mo}\left(\mathrm{NBu} \mathrm{t}^{\mathrm{A}} \mathrm{r}\right)_{3}$

Os trabalhos do grupo de Shilov $22,26,41$ são bons exemplos da química da fixação de $\mathrm{N}_{2}$. $\mathrm{O}$ aspecto único do trabalho de Shilov foi o desenvolvimento de sistemas que fixam $\mathrm{N}_{2}$ em água/metanol (2-10\% de água). Hidróxidos de $\mathrm{Mo}^{\mathrm{III}}, \mathrm{V}^{\mathrm{II}}$, $\mathrm{Ti}^{\mathrm{II}}, \mathrm{Nb}^{\mathrm{III}}$ e $\mathrm{Ta}^{\mathrm{III}}$ apresentam uma intensa atividade catalítica quando recém preparados na mistura de solventes. Este constitui um dos modelos funcionais mais simples e mais eficientes da nitrogenase, reduzindo $\mathrm{N}_{2}$ até amônia, produzindo hidrazina intermediariamente. A reação é rápida à temperatura ambiente e também a baixas temperaturas, apresentando rendimentos de até $65 \%$. Estes são sistemas heterogêneos e combinam potenciais de redução negativos com configurações $\mathrm{d}^{2}$ ou d $\mathrm{d}^{3}$, e alguns são genuinamente catalíticos, embora os mecanismos definitivos nunca tenham sido determinados.

Shilov sugere que a condição importante para a redução do $\mathrm{N}_{2}$ é a formação de um complexo polinuclear com vários centros doadores, capazes de transferir pelo menos quatro elétrons em meio prótico. A estrutura exata do complexo polinuclear de $\mathrm{V}^{\mathrm{II}}$ na mistura $\mathrm{V}(\mathrm{OH})_{2} /$ $\mathrm{Mg}(\mathrm{OH})_{2}$ é desconhecida. Postula-se que os íons de vanádio são unidos por pontes $\mathrm{O}-\mathrm{Mg}-\mathrm{O}$, formando complexos binucleares do tipo VNNV como intermediários de reação $0^{41}$ (Figura 5).

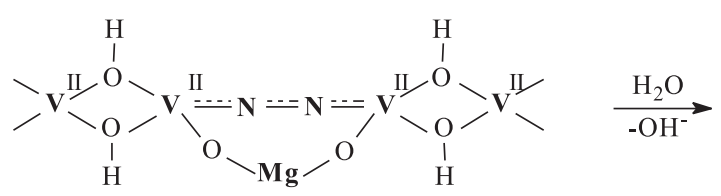

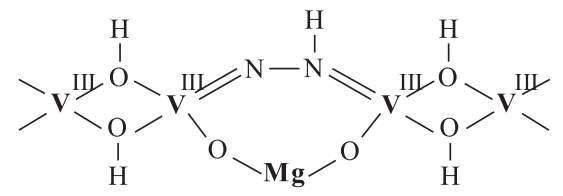<smiles>N#CC(N)O[SnH3]</smiles>

Figura 5. Espécies polinucleares propostas por Shilov na fixação de $N_{2}$ por géis de hidróxido de vanádio(II) 
A necessidade do desenvolvimento de um sistema de catálise homogênea levou Shilov à descoberta de que complexos de $\mathrm{V}^{\mathrm{II}}$ com catecóis são capazes de reduzir $\mathrm{N}_{2}$ em água e em soluções alcoólicas, com um rendimento de até $75 \%$ (em metanol), tendo amônia como único produto ${ }^{22}$. Estudos de EPR de soluções contendo $\mathrm{V}^{\mathrm{II}} \mathrm{e}$ catecol em metanol, na presença de diferentes concentrações de $\mathrm{LiOCH}_{3}$ e $\mathrm{NaOCH}_{3}$, indicaram a presença de complexos trinuclares com uma estrutura flexível o suficiente para permitir a formação de uma ponte $\mathrm{N}-\mathrm{N}$ entre dois átomos de vanádio (Figura 6). A etapa determinante do mecanismo de redução do $\mathrm{N}_{2}$ coordenado envolveria a interação de dois complexos trinucleares, formando um complexo tetranuclear $\mathrm{V}_{4} \mathrm{~N}_{2}$, e um dímero de $\mathrm{V}^{\mathrm{II}}$, que seria responsável pela produçao de $\mathrm{H}_{2}{ }^{37}$.
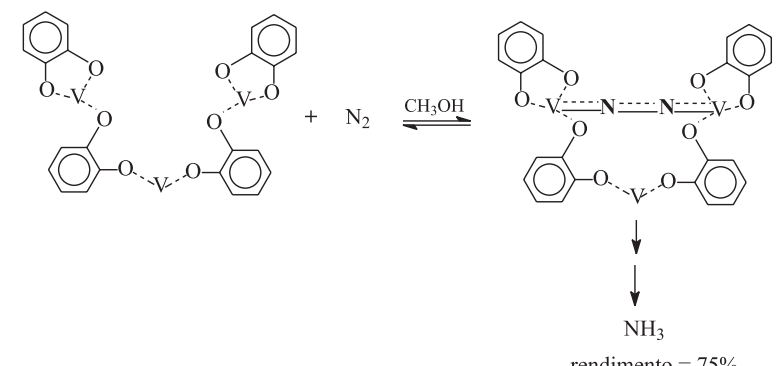

Figura 6. Intermediários polinucleares de catecóis de vanádio(II) formados na redução de $\mathrm{N}_{2}$ e propostos por Shilov

O trabalho de Shilov impulsionou fortemente a química de complexos trinucleares de vanádio. Os relatos são relativamente recentes, e dados sobre sua reatividade são praticamente inexistentes. Os complexos mais conhecidos contêm a unidade central $\left\{\mathrm{V}_{3} \mathrm{O}\right\}$ rodeada por seis carboxilatos bidentados e em ponte, como os originalmente descritos por Glowiak ${ }^{56}$. Leigh descreveu a preparação de $\left[\mathrm{M}_{3}(\mu-\right.$ Cl $\left.)_{3}\left(\mu_{3}-\mathrm{Cl}\right)_{2}(\text { tmeda })_{3}\right]^{+}$, onde $\mathrm{M}=\mathrm{Ti}, \mathrm{V}, \mathrm{Fe}, \mathrm{Co}$ e $\mathrm{Ni}$, e tmeda $=$ $N, N, N$ ', $N$ '-tetrametiletilenodiamina ${ }^{57}$, sendo a estrutura cristalina ${ }^{38}$ e as propriedades espectrais ${ }^{39}$ do complexo $\left[\mathrm{V}_{3} \mathrm{Cl}_{5}(\mathrm{tmeda})_{3}\right]\left(\mathrm{BPh}_{4}\right)$ conhecidas. Mais recentemente, a síntese e a caracterização estrutural do primeiro complexo trinuclear heterobimetálico contendo vanádio(II) e ferro(II) do tipo $\left.\left[\mathrm{V}_{\mathrm{x}} \mathrm{Fe}_{3-\mathrm{x}}(\mu-\mathrm{Cl})_{3}\left(\mu_{3}-\mathrm{Cl}\right)_{2} \text { (tmeda) }\right]_{3}\right]^{+}$foram descritas ${ }^{40}$. Nenhum destes compostos, no entanto, apresentaram capacidade de coordenação ou de redução de $\mathrm{N}_{2}$.

Gambarotta $^{58}$ isolou o primeiro composto homobimetálico de vanádio contendo $\mathrm{N}_{2}$ coordenado, [ $\left.\left\{\left(2-\mathrm{Me}_{2} \mathrm{NCH}_{2} \mathrm{C}_{6} \mathrm{H}_{4}\right)_{2} \mathrm{VPy}\right\}_{2}\left(\mu-\mathrm{N}_{2}\right)\right]$ (Figura 7) através da reação entre $\left[\mathrm{VCl}_{2}\right.$ (tmeda) ${ }^{2}$ (tmeda $=N, N, N^{\prime}, N^{\prime}-$ tetrametiletilenodiamina) e $2-\mathrm{Li}\left(\mathrm{CH}_{2} \mathrm{NCH}_{3}\right)_{2} \mathrm{C}_{6} \mathrm{H}_{4}$ na presença de piridina, confirmando que $\mathrm{V}$ (II) é suficientemente reativo para interagir com $\mathrm{N}_{2}$. Em complexos trinucleares de samário(III) com o

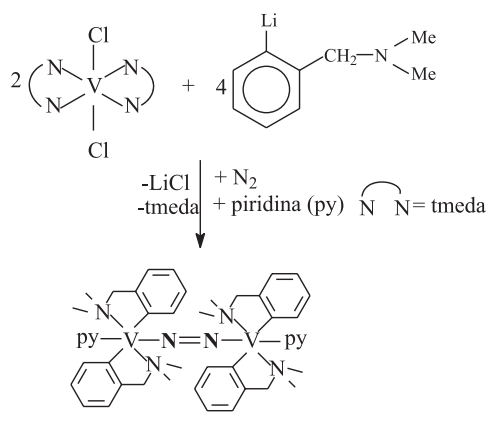

Figura 7. Primeiro complexo isolado de vanádio(II) contendo dinitrogênio coordenado: [\{2-Me $\left.\left.\left.\mathrm{NCH}_{2} \mathrm{C}_{6} \mathrm{H}_{4}\right)_{2} \mathrm{VPy}\right\}_{2}\left(\mu-\mathrm{N}_{2}\right)\right]$ ligante tetraaza calix-tetrapirrol, Gambarotta ${ }^{55}$ descreveu a redução por quatro elétrons da molécula de $\mathrm{N}_{2}$ coordenado, observando-se distâncias N-N de 1,502(5) e 1,525(4) ̊.

A importância da polinuclearidade para a ativação da molécula de $\mathrm{N}_{2}$ também foi demonstrada por Ferguson ${ }^{43}$, na produção de hidrazina e amônia a partir da protonação do complexo binuclear de vanádio(II) $\left[(\mathrm{Mes})_{3} \mathrm{~V}\left(\mu-\mathrm{N}_{2}\right) \mathrm{V}(\mathrm{Mes})_{3}\right]$, Mes $=2,4,6-\mathrm{Me}_{3} \mathrm{C}_{6} \mathrm{H}_{2}$.

$\mathrm{O}$ desenvolvimento de complexos como $\left[\mathrm{H}_{2} \mathrm{Fe}(\mathrm{CO})_{4}\right]$ e $\left[\mathrm{ZrHCl}\left(\eta^{5}-\mathrm{C}_{5} \mathrm{H}_{5}\right)\right]^{35}$ capazes de agir como fontes de hidrogênio para protonar o $\mathrm{N}_{2}$, ao invés de um ácido mineral, é uma estratégia bastante interessante e relativamente recente ${ }^{59}$. As dificuldades nas sínteses destes tipos de compostos foram significativamente superadas quando Hidai ${ }^{35,52}$ descobriu que o complexo $\left[\mathrm{RuCl}\left(\mathrm{Ph}_{2} \mathrm{PCH}_{2}\right.\right.$ $\left.\left.\mathrm{CH}_{2} \mathrm{PPh}_{2}\right)_{2}\right]^{+}$reage reversivelmente com $\mathrm{H}_{2}$, produzindo o complexo $\left[\mathrm{RuCl}\left(\eta^{2}-\mathrm{H}_{2}\right)\left(\mathrm{Ph}_{2} \mathrm{PCH}_{2} \mathrm{CH}_{2} \mathrm{PPh}_{2}\right)_{2}\right]^{+}$. Hidai ${ }^{25}$ investigou a reatividade do $\mathrm{N}_{2}$ coordenado a metais em complexos do tipo $\left[\mathrm{M}\left(\mathrm{N}_{2}\right)_{2}(\mathrm{~L})_{4}\right](\mathrm{M}=$ Mo ou W e $\mathrm{L}=$ fosfina terciária). $\mathrm{O}$ tratamento de cis- $\left[\mathrm{W}\left(\mathrm{N}_{2}\right)_{2}\right.$ $\left.\left(\mathrm{PMe}_{2} \mathrm{Ph}\right)_{4}\right]$ com excesso de trans- $\left[\mathrm{RuCl}\left(\eta^{2}-\mathrm{H}_{2}\right)(\text { difosfina })_{2}\right]^{+}$sob atmosfera de $\mathrm{H}_{2}(1 \mathrm{~atm})$ e condições controladas de temperatura $\left(55^{\circ} \mathrm{C}\right)$ chega a rendimentos totais acima de $70 \%$ de amônia, e traços de hidrazina, dependendo da difosfina utilizada e do contra-íon do complexo de diidrogênio ${ }^{35}$ (Figura 8). Nestes sistemas, apenas um íon hidrogênio, formado pela clivagem heterolítica da molécula de $\mathrm{H}_{2}$ coordenada ao rutênio, é utilizado para formar a ligação N-H, de forma que são necessários pelo menos três equivalentes do complexo de diidrogênio para formar um equivalente de amônia. $\mathrm{O}$ hidreto remanescente não é utilizado para formar uma nova ligação $\mathrm{N}-\mathrm{H}$. Além disso, todos os elétrons requeridos para a formação de $\mathrm{NH}_{3}$ são fornecidos pelo tungstênio, que não é regenerado após sua oxidação a $\mathrm{W}^{\mathrm{VI}}$.

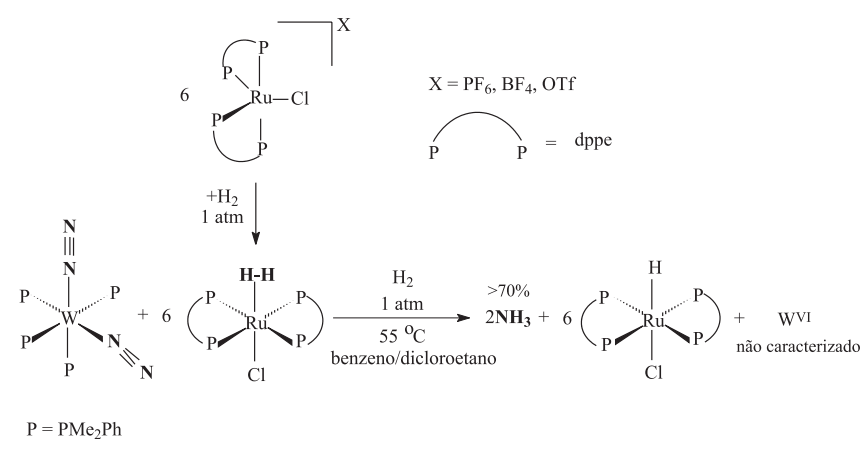

Figura 8. Síntese de amônia por reação entre os compostos-modelo de Hidai. A fonte de prótons é o diidrogênio-complexo de rutênio(II) e não o solvente

Leigh $^{23}$ também realizou estudos cinéticos com complexos deste tipo contendo Fe, Ru e Os. Foram estudadas reações de substituição de diidrogênio e dinitrogênio em complexos do tipo $[\mathrm{FeH}(\mathrm{L})$ (difosfina) $)_{2}^{+}\left(\mathrm{L}=\mathrm{H}_{2}\right.$ ou $\left.\mathrm{N}_{2}\right)$ (difosfina $=\mathrm{R}_{2} \mathrm{PCH}_{2} \mathrm{CH}_{2} \mathrm{PR}_{2}(\mathrm{R}=\mathrm{Et}$ ou $\mathrm{Me})$ ou $\mathrm{P}\left(\mathrm{CH}_{2} \mathrm{CH}_{2} \mathrm{PR}_{2}\right)_{3}\left(\mathrm{R}^{\prime}=\mathrm{Me}\right.$ ou $\left.\mathrm{Ph}\right)$ por ligantes L' $(\mathrm{MeCN}$, $\mathrm{PhCN}$ ou $\mathrm{Cl}^{-}$), em acetona ou thf. A determinação dos parâmetros de ativação levaram a uma proposta mecanística para estas reações, em que a perda de $\mathrm{H}_{2} / \mathrm{N}_{2}$ pelo diidrogênio/dinitrogênio complexo é determinante na velocidade do processo, seguida pela rápida coordenação de L'.

Estes estudos levantam a questão se não seria $\mathrm{o}_{2}$ a fonte de elétrons para a redução de $\mathrm{N}_{2}$ no meio biológico, ao mesmo tempo em que aproximam a química dos complexos de dinitrogênio da catálise Haber-Bosch.

Pickett ${ }^{36}$ descreveu a redução de $\mathrm{N}_{2}$ em meio alcoólico (metanol, etanol ou isopropanol) sob condições brandas e controladas, geran- 
do amônia e um nitreto-complexo a partir do complexo trans$\left[\mathrm{W}\left(\mathrm{N}_{2}\right)_{2}\left(\mathrm{Ph}_{2} \mathrm{PCH}_{2} \mathrm{CH}_{2} \mathrm{PPh}_{2}\right)_{2}\right]$. Neste caso, o ciclo catalítico foi completado através de uma redução eletroquímica. Mais recentemente, o autor propôs ${ }^{36}$ mecanismos de redução de $\mathrm{N}_{2}$ no sítio ativo da nitrogenase com base na redução, também eletroquímica, do diazenocomplexo $\left[\mathrm{Mo}_{2} \mathrm{Cp}_{2}(\mu-\mathrm{SMe})_{3}\left(\mu-\eta^{2}-\mathrm{H}-\mathrm{N}=\mathrm{N}-\mathrm{R}\right)\right]^{+}$.

Em contraste com a inabilidade de redutores mais fortes como $\mathrm{Na}$, K ou Cs, estes resultados demonstram definitivamente a capacidade de complexos metálicos em ligar $\mathrm{N}_{2}$, quebrar a tripla ligação e promover a hidrogenólise da ligação M-N.

\section{REAÇÕES DE INSERÇÃO DE DINITROGÊNIO E A QUÍMICA ORGANOMETÁLICA DAS NITROGENASES}

Complexos de dinitrogênio podem sofrer reações de alquilação ou protonação de um átomo de nitrogênio terminal, permitindo a síntese de moléculas orgânicas sofisticadas. Excelentes resultados neste sentido têm sido descritos pelo grupo de Hidai ${ }^{52,60}$ e por Mori ${ }^{61}$, observando-se várias transformações úteis em síntese orgânica ( $\mathrm{Fi}$ gura 9). Estas descobertas são muito importantes porque demonstram que é realmente possível usar $\mathrm{N}_{2}$ como material de partida. A conversão destes processos em ciclos catalíticos é o grande desafio para torná-los comercialmente viáveis.

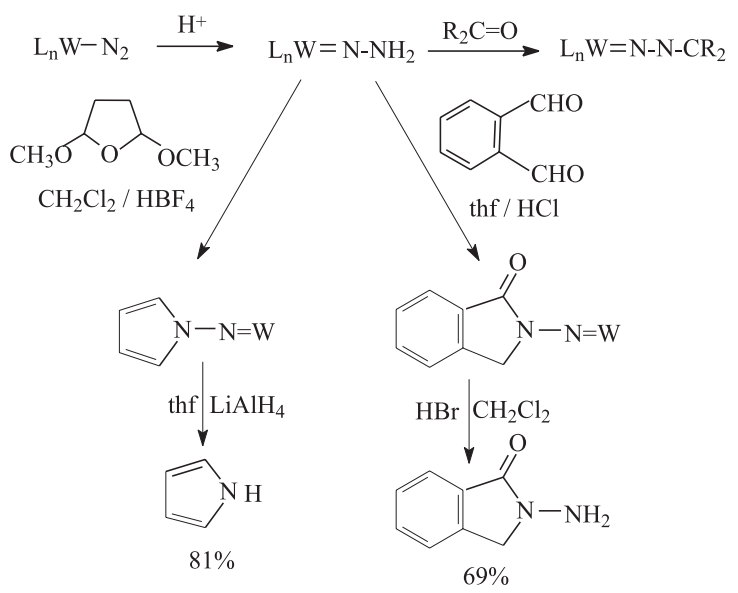

Figura 9. Exemplos de reações de organofuncionalização de dinitrogêniocomplexos

$\mathrm{Na}$ busca por modelos competitivos para a nitrogenase, encontram-se descritas reações com outros substratos além do $\mathrm{N}_{2}^{59,62,63}$. As nitrogenases podem, por exemplo, reduzir acetileno a etileno e ciclopropeno a ciclopropano e propeno. Os modelos para explicar esta atividade da enzima baseiam-se na complexação do hidrocarboneto seguida de protonação. Os modelos clássicos são hidretocomplexos de molibdênio(0), [ $\left.\mathrm{MoH}_{4}\left(\mathrm{Ph}_{2} \mathrm{PCH}_{2} \mathrm{CH}_{2} \mathrm{PPh}_{2}\right)_{2}\right]$, $\left[\mathrm{MoH}_{4}\left(\mathrm{PMePh}_{2}\right)_{4}\right]^{64}$ e de ferro(II), $\left[\mathrm{FeH}\left(\mathrm{H}_{2}\right)(\mathrm{dmpe})_{2}\right]^{+62,64,65}$, cuja química foi revista por Henderson ${ }^{59} \mathrm{e} \mathrm{Leigh}^{62}$. Em geral, os produtos da protonação de ciclopropeno na presença de complexos de metais de transição são diversos e dependem não apenas do tipo de metal, mas também do ácido e do solvente usados. Isto torna a interpretação da atividade da nitrogenase ainda mais difícil. As evidências sugerem que a redução ocorre através da complexação do substrato seguida do fluxo de prótons e elétrons, tal que a protonação do metal precede a transferência para o hidrocarboneto.

\section{COMPLEXOS-MODELO PARA A LIGAÇÃO DO N ${ }_{2}$ NAS NITROGENASES}

Um bom exemplo da capacidade de coordenação de $\mathrm{N}_{2}$ a com- plexos metálicos é encontrado nos trabalhos de Fryzuk ${ }^{5,51}$. Complexos de zircônio(II) com ligantes amideto-fosfinas tri- e tetradentados (gerados in situ) reagem com $\mathrm{N}_{2}$, formando complexos binucleares com ligações "side on", como mostrado na Figura 10. Um destes complexos possui a maior distância N-N jamais medida $[1,548(7) \AA$ versus $1,098(1) \AA$ do $\mathrm{N}_{2}$ livre], indicando uma redução de quatro elétrons. Estes compostos reagem com $\mathrm{H}_{2}(\mathrm{~g})$, resultando em uma hidrogenação parcial.

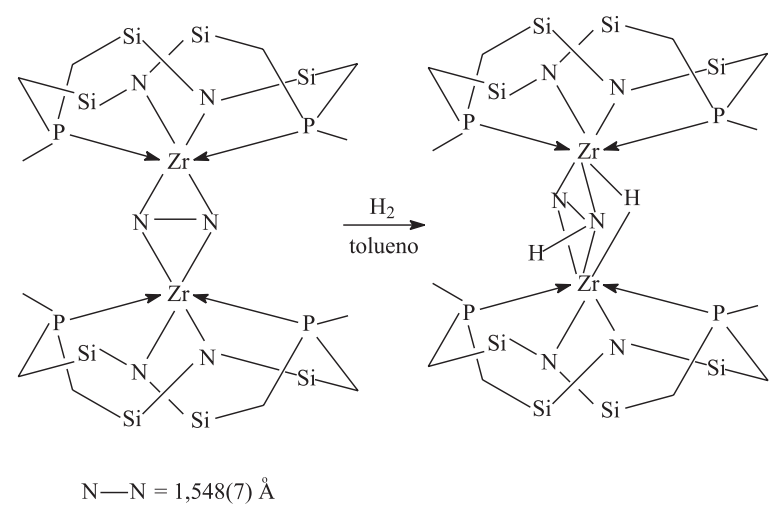

Figura 10. Complexos de zircônio(II) e dinitrogênio com ligantes amidetofosfinas com a maior distância $N-N$ medida

Sharp ${ }^{54}$ recentemente demonstrou a auto-montagem do cluster $\left[(\mathrm{LAu})_{6}\left(\mathrm{~N}_{2}\right)\right]^{2+}, \mathrm{L}=\mathrm{PPh}_{2} \mathrm{Me}$ dentre outras, a partir da reação de duas subunidades trinucleares de ouro com hidrazina. O produto contém $\mathrm{N}_{2}$ atuando como ponte entre os seis átomos de ouro, em uma estrutura semelhante a uma gaiola (Figura 11). O cluster decompõe-se em meio ácido com protonação completa ou parcial e rendimentos de 13 a $100 \%$ de $\mathrm{NH}_{3}$, dependendo da natureza do ligante auxiliar (fosfina), demonstrando que a ligação de $\mathrm{N}_{2}$ a seis átomos de ouro pode facilitar a clivagem da ligação N-N. A distância de ligação N-N no cluster é de 1,457(14) $\AA$, indicando uma ligação simples. A separação entre os dois átomos de ouro é muito grande, maior que 3,6 $\AA$, mostrando claramente que a estrutura do cluster hexanuclear é mantida graças à molécula de $\mathrm{N}_{2}$ em ponte.

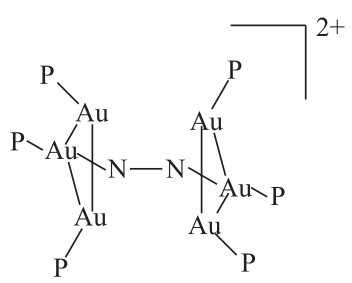

$$
\begin{aligned}
& \mathrm{P}=\mathrm{PPh}_{2}{ }^{\mathrm{iPr}} \\
& \text { contra-íon }=\mathrm{BF}_{4}{ }^{-} \\
& \mathrm{N}-\mathrm{N}=1,475(14) \AA
\end{aligned}
$$

Figura 11. Cluster obtido pela reação: $2\left[\left(\mathrm{PPh}_{2}{ }^{i} \mathrm{PrAu}\right)_{3}(\mu-\mathrm{O})\right]^{+}+\mathrm{NH}_{2} \mathrm{NH}_{2}$ $\rightarrow\left[\left(\mathrm{PPh}_{2}{ }^{i} \mathrm{PrAu}\right)_{6}\left(\mathrm{~N}_{2}\right)_{2}\right]^{2+}+2 \mathrm{H}_{2} \mathrm{O}$, demonstrando que a ligação de $\mathrm{N}_{2}$ a seis átomos de ouro pode facilitar a clivagem da ligação $N-N$

Será esta a forma como o $\mathrm{N}_{2}$ se liga nas nitrogenases? Esta proposta está de acordo com a recente descoberta do nitrogênio intersticial no cofator da nitrogenase de molibdênio. A grande distância de separação entre dois átomos de molibdênio no síto ativo da FeMo nitrogenase é desfavorável, no entanto, a esta hipótese. O mesmo argumento aplica-se se considerarmos a participação de um sistema heterobimetálico, Mo- $\mathrm{N}_{2}$-Fe A proposta mais aceita atualmente é a de que o $\mathrm{N}_{2}$ se liga ao heteroátomo (Mo ou V) e que os elétrons para a redução são transferidos para o $\mathrm{N}_{2}$ através dos clusters $\mathrm{P}$, os prótons tendo origem no solvente, $\mathrm{H}_{2} \mathrm{O}^{7}$. 
Inúmeros trabalhos sobre a modelagem da nitrogenase através da realização de cálculos teóricos estão descritos ${ }^{11}$. Os modelos descrevem prováveis "turn-over states" para o cofator FeMoco e o modo de ligação com os substratos, bem como possíveis mecanismos de redução centrados tanto no ferro, como no molibdênio.

Os modelos de ligação existentes, no entanto, não permitem racionalizar o fato de que o interior do cofator FeMoco é grande o bastante para acomodar o $\mathrm{N}_{2}$, mas não os intermediários de redução, como $\mathrm{N}_{2} \mathrm{H}_{4}$ ou duas moléculas de amônia.

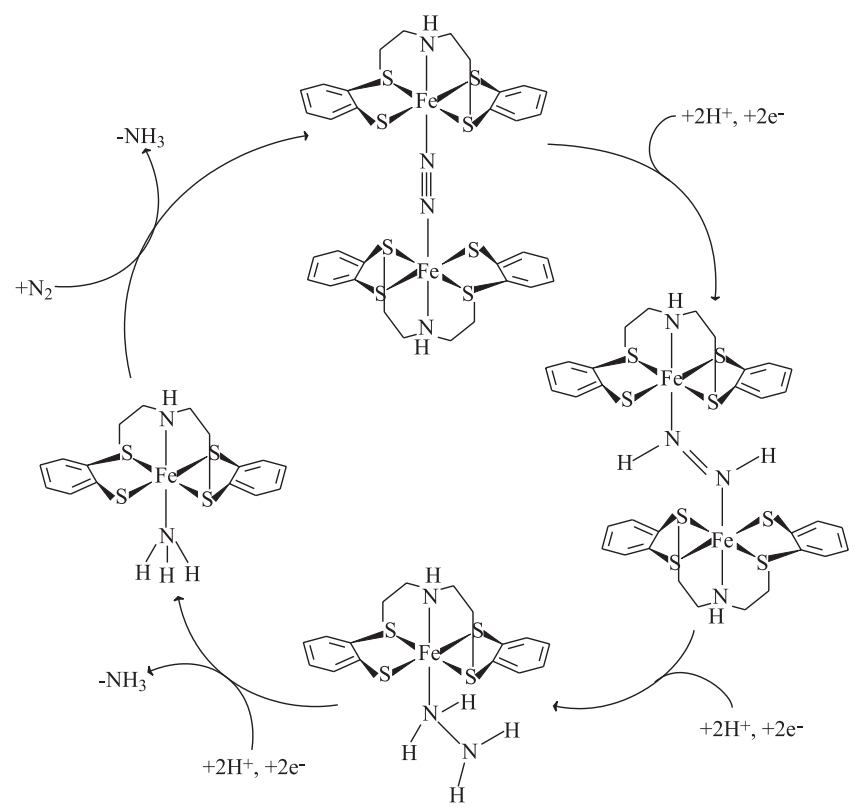

Figura 12. Ciclo catalítico hipotético para a fixação de $N_{2}$ pelo fragmento [Fe( ' $\mathrm{N}_{\mathrm{H}} \mathrm{S}_{4}$ ')]

Sellmann e Sutter ${ }^{33}$ apresentaram uma nova alternativa baseada na quebra de uma das ligações Fe-S-Fe do sítio ativo da enzima. Este modelo surgiu após a investigação de complexos-modelo contendo ligantes organosulfurados ${ }^{11,53}$. Os resultados mais significativos foram obtidos com complexos de ferro. Os autores prepararam o diazeno-complexo $\left[\mu-\mathrm{N}_{2} \mathrm{H}_{2}\left\{\mathrm{Fe}\left({ }^{\circ} \mathrm{N}_{\mathrm{H}} \mathrm{S}_{4} \text { ') }\right\}_{2}\right]\right.$ e propuseram um ciclo catalítico hipotético para a fixação de $\mathrm{N}_{2}$ (Figura 12$)^{66}$. No ciclo, $\mathrm{N}_{2}$ liga-se ao fragmento $\left[\mathrm{Fe}\left({ }^{\prime} \mathrm{N}_{\mathrm{H}} \mathrm{S}_{4}\right.\right.$ ') $)$ e o complexo resultante é reduzido até o amim-complexo, passando pelos complexos contendo os intermediários da redução, $\mathrm{N}_{2} \mathrm{H}_{2}$ e $\mathrm{N}_{2} \mathrm{H}_{4}$. Não há evidências experimentais para a existência do complexo de dinitrogênio, no entanto, os complexos de $\mathrm{NH}_{3}, \mathrm{~N}_{2} \mathrm{H}_{2}$ e $\mathrm{N}_{2} \mathrm{H}_{4}$ foram sintetizados e completamente caracterizados, em particular, o diazeno-complexo ${ }^{66,67}$. O estado de baixo spin do ferro(II) é imposto por restrições estéreas do ligante ' $\mathrm{N}_{\mathrm{H}} \mathrm{S}_{4}$ ', uma característica possivelmente garantida pela cadeia protéica da nitrogenase. A labilidade da ligação $\mathrm{Fe}-\mathrm{S}$ e a basicidade de Brönsted também foi observada no modelo sintético, conferindo reversibilidade para as reações de dissociação, bem como um fluxo de prótons e elétrons ${ }^{68}$. Estas observações permitiram vislumbrar um estado ativado ("turn-over state") para o cofator FeMoco, onde uma ponte Fe-S-Fe é aberta permitindo a coordenação de aminoácidos e moléculas de água vizinhas aos centros de ferro. Isto daria origem a dois centros de ferro(II) pentacoordenados, como ilustrado na Figura 13, que poderiam ligar $\mathrm{N}_{2}$ com a subseqüente redução acoplada ao fluxo de prótons. Este modelo prevê ainda que os heteroátomos, Mo ou V, agiriam apenas como moduladores do potencial redox da enzima.

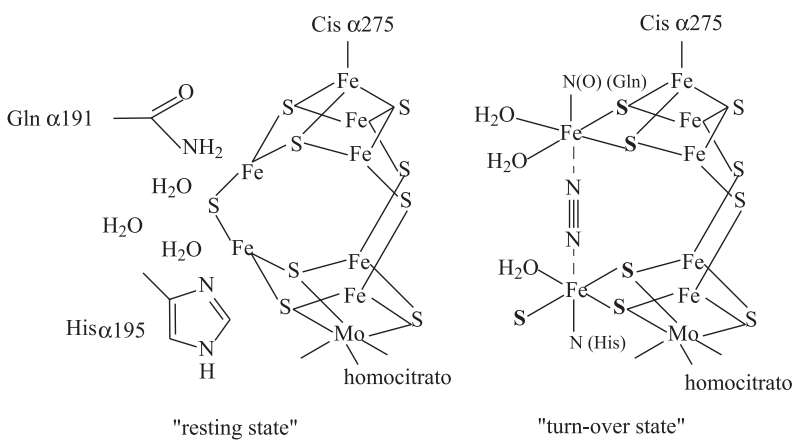

Figura 13. Proposta de abertura do cofator FeMoco após redução de dois elétrons e formação de dois centros de ferro(II) baixo-spin pentacoordenados

\section{CONSIDERAÇÕES FINAIS}

Sintetizando os avanços mais relevantes sob o ponto de vista da modelagem da nitrogenase, destacamos os trabalhos de Shilov, Gambarotta, Fryzuk, Cummins, Sharp, Sellmann e Hidai que apontam para a necessidade da interação cooperativa de vários metais para a obtenção de complexos robustos de $\mathrm{N}_{2}$ e reduções significativas de $\mathrm{N}_{2}$. A redução catalítica da amônia é possível em condições brandas. Quanto mais extensa a ativação e a funcionalização da molécula de $\mathrm{N}_{2}$, mais a ligação M-N se aproxima de uma função M-amideto ou nitreto. Com metais de transição do início da série e lantanídeos, estas unidades M-amideto são particularmente estáveis e quebradas apenas sob condições hidrolíticas. Estes resultados são pouco atrativos para a construção de ciclos catalíticos. A questão principal é balancear a estabilidade das ligações M-H e M-C com a M-N. Um processo catalítico necessariamente requer a labilização da molécula funcionalizada resultante da ativação do $\mathrm{N}_{2}$, para permitir a regeneração do complexo em baixo estado de oxidação. Neste sentido, há pouca esperança para os metais do início da série. Além disso, observa-se que a inércia da ligação $\mathrm{M}-\mathrm{N}_{2}$ aumenta com a capacidade de retrodoação do metal. Em geral, complexos de $\mathrm{N}_{2}$ mais estáveis são obtidos com metais ricos em elétrons e em baixo estado de oxidação. Complexos de dinitrogênio com metais do início da série de transição são tipicamente lábeis, e a labilidade aumenta com o aumento do número de oxidação. Portanto, a química dos metais de transição do meio para a direita da tabela periódica deve ser mais promissora.

Descobertas importantes relacionadas ao uso de $\mathrm{N}_{2}$ como material de partida viável e a elaboração de estruturas moleculares complexas, como nos trabalhos de Hidai, foram obtidas em poucos casos.

A recente descoberta do grupo de Rees sobre o átomo de nitrogênio intersticial no cofator FeMoco proporcionará uma nova era de possibilidades, para os químicos sintéticos, para a ativação de $\mathrm{N}_{2}$. Os resultados obtidos até o momento são muito interessantes porque provam a viabilidade do uso de dinitrogênio como material de partida mas, na sua maioria, sem catálise. Por outro lado, a criação de ciclos catalíticos viáveis requer a inserção direta de $\mathrm{N}_{2}$ em ligações reativas como M-H ou M-C.

As tentativas de modelagem da estrutura e da reatividade do cofator através de métodos teóricos deverão ser reconsideradas face à presença do nitrogênio intersticial e dos seus efeitos sobre a estrutura e o estado de oxidação dos átomos de ferro. O desafio continua.

\section{AGRADECIMENTOS}

Ao PRONEX (fase 1), FUNPAR e CNPq pelo suporte financeiro. Ao Prof. Dr. F. de O. Pedrosa, Depto. de Bioquímica - UFPR, pelo apoio na formação do nosso grupo de pesquisa. 


\section{REFERÊNCIAS}

1. Eady, R. R.; Leigh, G. J.; J. Chem. Soc., Dalton Trans. 1994, 2739.

2. Kim, J. S.; Rees, D. C.; Biochemistry 1994, 33, 389; Kim, J. S.; Rees, D. C.; Science 1992, 257, 1677; Peters, J. W.; Stowell, M. H. B.; Soltis, S. M.; Finnegan, M. G.; Johnson, M. K.; Rees, D. C.; Biochemistry 1997 36, 1181; Mayer, S. M.; Lawson, D. M.; Gormal, C. A.; Roe, S. M.; Smith, B. E.; J. Mol. Biol. 1999, 292, 871.

3. Leigh, G. J.; Science 1998, 279, 506

4. Howard, J. B.; Rees, D. C.; Chem. Rev. 1996, 96, 2965; Burgess, B. K.; Lowe, D. J.; Chem. Rev. 1996, 96, 2983; Lawson, D. M.; Smith, B. E.; Metal Ions in Biological Systems 2002, 39, 75.

5. Fryzuk, M. D.; Johnson, S. A.; Coord. Chem. Rev. 2000, 200-202, 379.

6. Bortels, H.; Arch. Mikrobiol. 1930, 1, 333; Bortels, H.; Zentralbl. Bakteriol. Parasitenkd. Infektionskr. Abt. 1936, 2, 193.

7. Leigh, G. J.; Science 1995, 268, 827.

8. Vol'pin, M. E.; Schur, V. B.; Dokl. Acad. Nauk. 1964, 156, 1102.

9. Allen, A. D.; Senoff, C. V.; Chem. Commun. 1965, 621.

10. Eady, R. R.; J. Inorg. Biochem. 2001, 86, 42; Eady, R. R.; Chem. Rev. 1996, 96, 3013.

11. Lovell, T.; Li, J.; Case, D. A.; Noodleman, L.; J. Am. Chem. Soc. 2002, 124, 4546; Reiher, M.; Salomon, O.; Sellmann, D.; Hess, B. A.; Chem. -Eur. J. 2001, 7, 5195; Reiher, M.; Sellmann, D.; Hess, B. A.; Theor. Chem Acc. 2001, 106, 379; Lovell, T.; Li, J.; Liu, T. Q.; Case, D. A.; Noodleman, L.; J. Am. Chem. Soc. 2001, 123, 12392; Durrant, M. C.; Inorg. Chem. Commun. 2001, 4, 60; Rod, T. H.; Norskov, J. K.; J. Am. Chem. Soc. 2000 $122,12751$.

12. Einsle, O.; Tezcan, F. A.; Andrade, S. L. A.; Schmid, B.; Yoshida, M.; Howard, J. B.; Rees, D. C.; Science 2002, 297, 1696.

13. Smith, B. E; Science 2002, 297, 1654

14. Maritano, S.; Fairhurst, S. A.; Eady, R. R.; J. Biol. Inorg. Chem. 2001, 6 , 590.

15. Chiu, H. J.; Peters, J. W.; Lanzilotta, W. N.; Ryle, M. J.; Seefeldt, L. C.; Howard, J. B.; Rees, D. C.; Biochemistry 2001, 40, 641.

16. Syrtsova, L. A.; Timofeeva, E. A.; Russ. Chem. Bull. 2001, 50, 1789.

17. Eady, R. R.; Robson, R. L.; Richardson, T. H.; Miller, R. W.; Hawkins, M.; Chem. Rev. 1987, 244, 197.

18. Rehder, D.; J. Inorg. Biochem. 2000, 133.

19. Ribbe, M.; Gadkari, D.; Meyer, O.; J. Biol. Chem. 1997, 272, 26627; Shestakov, A. F.; Shilov. A. E.; Russ. Chem. Bull. 2001, 50, 2054.

20. Siemann, S.; Schneider, K.; Drottboom, M.; Muller, A.; Eur. J. Biochem. 2002, 269, 1650.

21. Leigh, G. J.; New J. Chem. 1994, 18, 157.

22. Shilov, A. E.; J. Mol. Catal. A: Chem. 1987, 41, 221.

23. Helleren, C. A.; Henderson, R. A; Leigh, G. J.; J. Chem. Soc., Dalton Trans. 1999, 1213.

24. Malunowi, E.; Tyrlik, S.; J. Organomet. Chem. 1974, 72, 269.

25. Chatt, J.; Pearman, A. J.; Richards, R. L.; Nature 1972, 253, 39.

26. Bazhenova, T. A.; Shilov, A. E.; Coord. Chem. Rev. 1995, 144, 69.

27. Gambarotta, S.; J. Organomet. Chem. 1995, 500, 117.

28. Didenko, L. P.; Gavrilov, A. B.; Shilova, A. K.; Strelets, V. V.; Ysarev, V. N.; Shilov, A. E.; Makhanev, V. D.; Banerjee, A. K.; Pospishil; Nouv. J. Chem. 1986, 10, 584.

29. Frykuk, M. D.; Love, J. B.; Rettig, S. J.; Young, V. G.; Science 1997, 275, 1445.

30. Han, J.; Coucouvanis, D.; Inorg. Chem. 2002, 41, 2738.; Zhou, H. C.; Su, W. P.; Achim, C.; Rao, V. P.; Holm, R. H.; Inorg. Chem. 2002, 41, 3191 Malinak, S. M.; Coucouvanis, D.; Prog. Inorg. Chem. 2001, 49, 599; Han, J.; Beck, K.; Ockwig, N.; Coucouvanis, D.; J. Am. Chem. Soc. 1999, 121, 10448; Lang, J. P.; Kawaguchi, H.; Tatsumi, K.; Chem. Commun. 1999, 2315; Arikawa, Y.; Kawaguchi, H.; Kashiwabara, K.; Tatsumi, K.; Inorg. Chem. 1999, 38, 4549; Dance, I.; J. Chem. Soc., Chem. Commun. 1998 523; Tyson, M. A.; Coucovanis, D.; Inorg. Chem. 1997, 37, 3808; Yoo, S J.; Hu, Z.; Goh, C.; Bominaar, E. L.; Holm, R. H.; Münck, E.; J. Am. Chem. Soc. 1997, 119, 8732;

31. Roussel, P.; Scott, P.; J. Am. Chem. Soc. 1998, 120, 1070.

32. Evans, W. J. ; Ulibarri, T. A.; Ziller, J. W.; J. Am. Chem. Soc. 1988, 110, 6877.

33. Sellmann, D.; Sutter, J.; Acc. Chem. Res. 1997, 30, 460.

34. Laplaza, C. E.; Cummins, C. C.; Science 1995, $268,861$.

35. Nishibayashi, Y.; Wakiji, I.; Hirata, K.; DuBois, M. R.; Hidai, M.; Inorg. Chem. 2001, 40, 578; Nishibayashi, Y.; Takemoto, S.; Shotaro, I.; Hidai, M.; Inorg. Chem. 2000, 39, 5946; Hidai, M.; Takahashi, T.; Yokotake, I.; Uchida, Y.; Chem. Lett. 1980, 645.
36. Pickett, C. J.; Talarmin, J.; Nature 1985, 317, 652; Le Grand, N.; Muir, K. W.; Petillon, F. Y.; Pickett, C. J.; Schollhammer, P.; Talarmin, J.; Chem.-Eur. J. 2002, 8, 3115.

37. Luneva, N. P.; Moravsky, A. P.; Shilov, A. E.; Nouv. J. Chim. 1982, 6, 245.

38. Hughes, D. L.; Larkworthy, L. F.; Leigh, G. J.; McGarry, C. J.; Sanders, J. R.; Smith, G. J.; de Souza, J. S.; J. Chem. Soc., Chem. Commun. 1994, 2137.

39. Niedwieski, A. C; Leigh, G. J.; Soares, J. F.; Nunes, F. S.; Motta Neto, J. D.; Int. J. Quantum Chem. 2002, 88, 245; Niedwieski, A. C.; Hitchcock, P. B.; Motta Neto, J. D.; Wypych, F.; Leigh, G. J.; Nunes, F. S.; J. Braz. Chem. Soc. 2003, 14, 750.

40. Evans, D.J.; Hitchcock, P. B.; Hughes, D. L.; Leigh, G. J.; Nicholson, B. H.; Nunes, F. S.; Niedwieski, A. C.; Soares, J. F.; Inorg. Chim. Acta 2001, $319,147$.

41. Shilov, A. E.; Denisov, N. T.; Efimov, O. N.; Shuvalov, V. P.; Shuvalova, N. I.; Shilova, A. K.; Nature 1971, 231, 460; Zones, S. I.; Palmer, M. R; Palmer, J. C.; Doemeny, J. M.; Schrauzer, G. N.; J. Am. Chem. Soc. 1978, 100,2113

42. Berno, P.; Gambarotta, S.; Angew. Chem., Int. Ed. 1995, 34, 822; Berno, P. Gambarotta, S.; Angew. Chem.1995, 107, 871.

43. Ferguson, R.; Solari, E.; Floriani, C.; Osella, D.; Ravera, M.; Re, N.; ChiesiVilla, A.; Rizzoli, C.; J. Am. Chem. Soc. 1997, 119, 10104.

44. Nekola, H.; Wang, D.; Gruning, C.; Gatjens, J.; Behrens, A.; Rehder, D.; Inorg. Chem. 2002, 41, 2379.

45. Davies, S. C.; Durrant, M. C.; Hughes, D. L.; Richards, R. L.; Sanders, J. R.; J. Chem. Soc., Dalton Trans. 2000, 4694.

46. Leigh, G. J.; Gimenez-Tenorio, M.; J. Am. Chem. Soc. 1991, 113, 5862

47. Franke, O.; Wiesler, B. E.; Lehnert, N,; Nather, C.; Ksenofontov, V.; Neuhausen, J.; Tuczek, F.; Inorg. Chem. 2002, 41, 3491.

48. van Tamelen, E.; Acc. Chem. Res. 1970, 3, 361; Vol'pin, M. E.; Schur, V. B.; Kudryavtsev, R. V.; Prodayko, L. A.; J. Chem. Soc., Chem. Commun. 1968, 1038; Sobota, P.; Janas, Z.; Inorg. Chim. Acta 1981, 53, L11.

49. Tayebani, M.; Gambarotta, S.; Bensimon, C.; Organometallics 1998, 17, 4282.

50. Kawaguchi, H.; Matsuo, T.; Angew. Chem., Int. Ed. 2002, 41, 2792.

51. Fryzuk, M. D.; Haddad, T. S.; Retting, S. J.; J. Am. Chem. Soc. 1990, 112, 8185; Cohen, J. D.; Fryzuk, M. D.; Loher, T. M.; Mylvaganam, M.; Retting, S. J.; Inorg. Chem. 1998, 37, 112.

52. Nishibayashi, Y.; Iwai, S.; Hidai, M.; Science 1998, 279, 540

53. Sellman, D.; Engl, K.; Heinemann, F. W.; Sieler, J.; Eur. J. Inorg. Chem. 2002, 1079 .

54. Shan, H.; Yang, Y.; James, A. J.; Sharp, P. R.; Science 1997, 275, 1445.

55. Guan, J.; Dube, T.; Gambarotta, S.; Yap, G. P. A.; Organometallics 2000, 19, 4820; Jubb, J.; Gambarotta, S.; J. Am. Chem. Soc. 1994, 116, 4477.

56. Glowiak, T.; Kubiak, M.; Jezowska-Trzebiatowska, B.; Bull. Acad. Pol. Sci., Ser. Sci. Chim. 1977, 25, 359.

57. Handley, D. A.; Leigh, G. J.; Inorg. Chim. Acta 2001, 314, 14.

58. Edema, J. J. H.; Gambarotta, S.; Meetsma, A.; J. Am.Chem. Soc. 1989, 111, 6878.

59. Henderson, R. A.; J. Chem. Soc., Dalton Trans. 1995, 503; Jia, G.; Morris, R. H.; Schweitzer, C. T.; Inorg. Chem. 1991, 30, 594.

60. Nishibayashi, Y.; Iwai, S.; Hidai, M.; J. Am. Chem. Soc. 1998, 120, 10559; Hidai, M.; Mizobe, Y.; Chem. Rev. 1995, 95, 1115; Hidai, M.; Ishii, Y.; J. Mol. Catal. A: Chem. 1996, 107, 105.

61. Mori, M.; Hori, K.; Akashi, M.; Hori, M.; Sato, Y.; Nishida, M.; Angew. Chem., Int. Ed. 1998, 37, 5.

62. Leigh, G. J.; McMahon, C. N.; J. Organomet. Chem. 1995, 500, 219.

63. Bazhenova T. A.; Bazhenova, M. A.; Petrova, G. N.; Mironova, S. A.; Kinet. Catal. 2002, 43, 351; Benton, P. M. C.; Christiansen, J.; Dean, D. R.; Seefeldt, L. C.; J. Am. Chem. Soc. 2001, 123, 1822.

64. Hills, A.; Hughes, D. L.; Jimenez-Tenorio, M.; Leigh, G. J.; McGeary, C. A.; Rowley, A. T.; Bravo, M.; McKenna, C. E.; McKenna, M. -C.; J. Chem. Soc., Chem. Commun. 1991, 522.

65. McKenna, C. E.; McKenna, M. -C.; Huang, C. W.; Proc. Natl. Acad. Sci. U.S.A. 1979, 76, 4773.

66. Sellmann, D.; Utz, J.; Blum, N.; Heinemann, F. W.; Coord. Chem. Rev. 1999, 190, 607 .

67. Sellmann, D.; Soglowek, F.; Knoch, F.; Ritter, G.; Dengler, J.; Inorg. Chem. 1992, 31, 3711; Sellmann, D.; Soglowek, F.; Knoch, F.; Moll, M. J.; Angew. Chem., Int. Ed. 1989, 28, 1271.

68. Sellmann, D.; Hofmann, T; Knoch, F.; Inorg. Chim. Acta 1994, 224, 61; Sellmann, D.; Friedrich, H.; Knoch, F.; Z. Naturforsch., B: Chem. Sci. 1993, 48, 1675. 